Surgery for

Congenital

Heart Disease

\title{
Left ventricular remodeling in hearts with tricuspid atresia: Morphologic observations and possible basis for ventricular dysfunction after surgery
}

Maria Angelica Binotto, $\mathrm{MD}^{\mathrm{a}}$

Maria de Lourdes Higuchi, MD

Vera Demarchi Aiello, MD ${ }^{\mathrm{b}}$
From the Department of Pediatric Cardiology and Adult Congenital Heart Disease ${ }^{\mathrm{a}}$ and the Laboratory of Pathology, Heart Institute $(\mathrm{InCor})^{\mathrm{b}}$, University of São Paulo Medical School, São Paulo, Brazil.

Supported by the Fundação de Amparo a Pesquisa do Estado de São Paulo (FAPESP), grant 98/07636-6.

Received for publication Sept 23, 2002; revisions requested Oct 29, 2002; revisions received Nov 13, 2002; accepted for publication Dec 2, 2002.

Address for reprints: Maria Angelica Binotto, MD, Heart Institute (InCor), University of São Paulo Medical School, Pediatric Cardiology, Av. Higienopolis, 1048/ 86, São Paulo 01238-000, Brazil (E-mail: conangelica@incor.usp.br).

J Thorac Cardiovasc Surg 2003;126: 1026-32

Copyright $\odot 2003$ by The American Association for Thoracic Surgery

$0022-5223 / 2003 \$ 30.00+0$

doi:10.1016/S0022-5223(03)00696-2
Objectives: This study was undertaken to assess the pattern of left ventricular hypertrophy, the myocardial capillary network, and the extracellular matrix in hearts with tricuspid atresia.

Methods: We examined 32 hearts with tricuspid atresia and 27 normal hearts from control subjects with similar age and sex distribution. Wall thickness, inlet length, and outlet length were obtained from the left ventricle. Immunohistochemical staining for von Willebrand factor was used to label myocardial capillaries. By means of computer-assisted morphometry, the following data were obtained from the inlet, apex, and outlet of the left ventricle: transverse myocyte diameter, myocyte nuclear volume fraction, capillary volume fraction, interstitial fibrous volume fraction, and endocardial thickness.

Results: The wall thickness in affected hearts was not different from that in control hearts. The left ventricular outlet length was significantly greater in the malformed hearts $(P=.005)$. The myocyte diameter did not differ from that in control hearts. The capillary volume fraction was decreased in the malformed hearts $(P<.001)$. The interstitial fibrous deposition was greater in the malformed hearts at all sites analyzed $(P<.001)$. Fibrosis was greater in the inlet and apex $(P=.004)$ and also in the subendocardial half of the ventricular wall than in the subepicardial half. According to a logistic regression model, age was the only variable associated with the probability of occurrence of fibrosis.

Conclusions: Our findings in hearts with tricuspid atresia possibly represent chronically induced volume overload in the presence of ischemia, rather than a typical model of volume overload. A decreased capillary volume fraction may indicate a greater susceptibility to ischemia. Fibrous deposition probably occurs early in life.

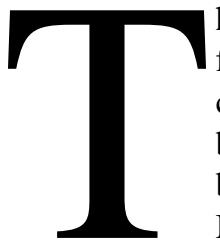

he application of the Fontan principle has improved survival and functional capacity for patients with univentricular atrioventricular connections. However, most centers have documented ongoing morbidity and mortality after surgery. ${ }^{1,2}$ Abnormal ventricular function, both systolic and diastolic, is clearly related to the late outcome. Myocardial hypertrophy is certainly recognized as a risk factor for the Fontan procedure. ${ }^{3}$ The systemic ventricle is chronically volume overloaded and 
subjected to longstanding hypoxemia and variable degrees of pressure overload, factors implicated in the myocardial remodeling process. In addition, the myocardium may be intrinsically abnormal, as has been suggested by Ho and colleagues $^{4}$ and, more recently, by Sanchez-Quintana and coworkers. ${ }^{5}$ In addition to the pathologic remodeling, expansion of cardiac mass during postnatal physiologic growth also takes place.

Few studies have addressed ventricular remodeling in hearts with univentricular atrioventricular connections. ${ }^{4-6}$ The purpose of this study was to analyze several aspects of the ventricular remodeling in hearts with tricuspid atresia, including myocardial hypertrophy, extracellular matrix deposition, and capillary network.

\section{Methods}

We studied 32 hearts with tricuspid atresia, obtained at autopsy from the pathology laboratory collection of the Heart Institute, University of São Paulo Medical School. The control group comprised 27 structurally normal hearts from children aged 1 day to 6 years who had died of non-cardiovascular-related diseases. Age and sex distributions were similar for the two groups (study group age $9.6 \pm 13.9$ months, median 2.5 months, 19 male and 13 female infants; control group age $9.6 \pm 17.4$ months, median 3 months, 15 male and 12 female infants). The specimens were inspected after a sequential segmental analysis. The following measurements were obtained from the left ventricle (LV): thickness of the free wall at the inlet (the level of the superior edge of the papillary muscles) and at the outlet (the same plane relative to the apex-base axis), inlet and outlet lengths (an estimation of cavity size), arterial valve diameters, and the major diameter of the ventricular septal defect (VSD). The VSD was considered restrictive when its major diameter was smaller than that of the arterial valve emerging from the rudimentary right ventricle. Transmural blocks of tissue were taken from the LV wall at the sites used for the measurements of wall thickness in the inlet and outlet and also from the apex of the LV. The tissue was processed routinely for histologic examination, and 5- $\mu \mathrm{m}$ sections were stained with hematoxylin and eosin and Masson trichrome stain. Histologic sections and staining considered to be technically inadequate were excluded from the analysis.

\section{Morphometric Analysis}

MYOCYTE TRANSVERSE DIAMETER. The morphometric measurements were carried out with an interactive computer-assisted image analyzer (Leica Quantimet; Leica Cambridge Ltd, United Kingdom). ${ }^{7}$ To avoid interobserver variation, a single investigator operated the analyzer.

The myocyte transverse diameter was measured at the level of the nucleus in the mesocardial layer of the ventricular wall, irrespective of the orientation of the section. Final magnification was $\times 400$.

In a pilot study, the evolution of the mean values and variance was analyzed to determine the number of measurements required. The mean values of myocyte diameter in 20, 40, 60, 80, 100, 120, 140,180 , and 200 observations were obtained. We opted to measure 60 myocytes per section.
CAPILLARY VOLUME FRACTION. Immunohistochemical staining for von Willebrand factor was used to label myocardial capillary endothelial cells. The stained inlet LV sections containing circumferentially oriented capillaries were imaged at a final magnification of $\times 1000$. For each section 30 random fields $11,456 \mu \mathrm{m}^{2}$ in area were analyzed. With computer-assisted morphometry and an interposed grid of 598 points, incident points on myocardial capillaries were counted. The proportion of the points lying over the structure of interest over the number of points in the grid was used as a measure of the volume fraction, or volume density, of myocardium occupied by that structure. ${ }^{8}$

NuClear VOlume FRACTION. With the same system described previously and an interposed grid of 598 points, incident points on myocyte nuclei were counted in sections of the LV inlet stained with hematoxylin and eosin. For each section 25 random fields were analyzed at the mesocardial layer of the ventricular wall.

QuANTIFication of Fibrous tissue. Fibrous content in the interstitial space was estimated by analyzing at least 10 microscopic fields in the subendocardial and subepicardial halves of the ventricular wall at a magnification of $\times 400$ (final resolution of 0.48 $\mu \mathrm{m} /$ pixel). Star-shaped scars and concentric perivascular fibrous tissue were excluded from the analysis. The interstitial fibrous volume fraction (IFVF) was expressed as a percentage of the myocardial area. Microscars were described qualitatively.

ENDOCARDIAL THICKNESS. Linear measurements of the endocardial thickness were obtained in histologic sections stained with hematoxylin-eosin from the inlet, apex, and outlet of the LV. A mean value was obtained from 10 measurements at each site.

\section{Statistical Analysis}

Values are given as means, SDs, and medians. Unpaired Student $t$ tests or Mann-Whitney tests as appropriate were used to compare values between control and study groups. Comparisons between subendocardial and subepicardial regions were done with the Wilcoxon test. The Friedman test was carried out for multiple comparisons, followed by the Tukey procedure as modified by Nemenyi. Correlation coefficients were obtained by the Spearman method. Logistic regression was used to identify independent predictors of the occurrence of fibrosis. Data were analyzed with SigmaStat 2.0 software (SPSS Inc, Chicago, Ill).

\section{Results}

\section{Heart Specimens}

The atrial arrangement was usual in all specimens. The ventriculoarterial connection was concordant in 20 hearts and discordant in 10. One of the remaining specimens showed a common arterial trunk emerging from the dominant LV, and another showed a double outlet from the LV. Twenty specimens showed signs of previous surgical intervention. Of these, 9 had a modified Blalock-Taussig shunt; 5 of them showed pulmonary artery banding, 3 with aortic coarctation repair. Of the remaining specimens, 5 had a Fontan-Kreutzer procedure (1 preceded by a modified Blalock-Taussig shunt and another by pulmonary artery banding) and 1 had undergone a Damus-Kaye-Stansel procedure associated with a bidirectional Glenn anastomosis. Death had occurred between 1 day and 2 years after surgery 
TABLE 1. Characteristics of patients

\begin{tabular}{|c|c|c|c|c|c|c|}
\hline Case & Sex & Age (mo) & $\begin{array}{l}\text { Ventriculoarterial } \\
\text { connection }\end{array}$ & Additional diagnosis & Previous operation & Interval* \\
\hline 1 & $\mathrm{M}$ & 0.1 & Discordant & Coarctation & None & - \\
\hline 2 & $\mathrm{M}$ & 0.2 & Concordant & TAPVC & None & - \\
\hline 3 & $\mathrm{M}$ & 0.2 & Concordant & Restrictive VSD, PS & MBTS & $5 d$ \\
\hline 4 & $\mathrm{~F}$ & 0.3 & Concordant & Restrictive VSD & MBTS & $6 d$ \\
\hline 5 & $\mathrm{~F}$ & 0.3 & Discordant & Restrictive VSD, coarctation & None & - \\
\hline 6 & $\mathrm{~F}$ & 0.4 & Concordant & Restrictive VSD, TAPVC & MBTS & $1 \mathrm{~d}$ \\
\hline 7 & $\mathrm{~F}$ & 0.5 & Concordant & Restrictive VSD, PS & MBTS & $2 d$ \\
\hline 8 & $M$ & 0.6 & Discordant & Coarctation & None & - \\
\hline 9 & $M$ & 0.7 & Concordant & Coarctation & None & - \\
\hline 10 & M & 0.9 & Concordant & $\begin{array}{l}\text { Restrictive VSD, pulmonary } \\
\text { atresia }\end{array}$ & None & - \\
\hline 11 & $\mathrm{~F}$ & 1 & Concordant & Pulmonary atresia & MBTS & $9 d$ \\
\hline 12 & M & 1.2 & Discordant & Coarctation & None & - \\
\hline 13 & $\mathrm{~F}$ & 2 & Discordant & Restrictive VSD, coarctation & None & - \\
\hline 14 & $\mathrm{~F}$ & 2 & Concordant & & PAB & $19 \mathrm{~d}$ \\
\hline 15 & M & 2 & Concordant & Restrictive VSD & None & - \\
\hline 16 & $\mathrm{~F}$ & 2.3 & Single outlet & Truncus arteriosus communis & None & - \\
\hline 17 & M & 2.7 & Discordant & PS & MBTS & $38 d$ \\
\hline 18 & M & 3 & Discordant & Coarctation & PAB plus coarctation repair & $16 d$ \\
\hline 19 & $\mathrm{~F}$ & 4 & Concordant & & None & - \\
\hline 20 & $M$ & 6 & Concordant & Restrictive VSD & Fontan-Kreutzer & $1 \mathrm{~d}$ \\
\hline 21 & $\mathrm{~F}$ & 6 & Concordant & Restrictive VSD & MBTS & $15 d$ \\
\hline 22 & $\mathrm{~F}$ & 7 & Concordant & & None & - \\
\hline 23 & $\mathrm{~F}$ & 8 & Double-outlet LV & Restrictive VSD, coarctation & PAB plus coarctation repair & $24 d$ \\
\hline 24 & $M$ & 8 & Discordant & Restrictive VSD, coarctation & PAB plus coarctation repair & $2 \mathrm{mo}$ \\
\hline 25 & $\mathrm{~F}$ & 9 & Concordant & PS & MBTS & $5 \mathrm{mo}$ \\
\hline 26 & $M$ & 24 & Discordant & Restrictive VSD & $\begin{array}{l}\text { Bidirectional Glenn anastomosis plus } \\
\text { Damus-Kaye-Stansel operation } \\
\text { plus mitral valve replacement }\end{array}$ & $3 d$ \\
\hline 27 & $\mathrm{M}$ & 26 & Concordant & PS & MBTS & $2 y$ \\
\hline 28 & $\mathrm{M}$ & 32 & Concordant & Restrictive VSD & Fontan-Kreutzer & $16 \mathrm{~d}$ \\
\hline 29 & $\mathrm{M}$ & 34 & Discordant & & $\mathrm{PAB}$ & $2.3 y$ \\
\hline 30 & $\mathrm{M}$ & 36 & Concordant & Restrictive VSD, PS & Fontan-Kreutzer & $12 d$ \\
\hline 31 & $\mathrm{M}$ & 36 & Concordant & & PAB, Fontan-Kreutzer & $3 \mathrm{mo}$ \\
\hline 32 & $\mathrm{M}$ & 50 & Concordant & Restrictive VSD & MBTS, Fontan-Kreutzer & $2 d$ \\
\hline
\end{tabular}

$\overline{T A P V C}$, Total anomalous pulmonary venous connection; $P S$, pulmonary valve stenosis; $M B T S$, modified Blalock-Taussig shunt; $P A B$, pulmonary artery banding.

*Interval from surgery to death.

TABLE 2. Gross measurements from the inlet and outlet of the LV, comparing hearts with tricuspid atresia and control hearts

\begin{tabular}{lllllll}
\hline & \multicolumn{2}{c}{ Tricuspid atresia } & & \multicolumn{2}{c}{ Control } & \\
\cline { 2 - 3 } & Mean \pm SD & Median & & Mean \pm SD & Median & $P$ value \\
\hline $\begin{array}{c}\text { Inlet wall } \\
\text { thickness }\end{array}$ & $0.68 \pm 0.17$ & 0.70 & & $0.69 \pm 0.22$ & 0.70 & .82 \\
$\begin{array}{c}\text { (cm) } \\
\text { Outlet wall } \\
\text { thickness }\end{array}$ & $0.70 \pm 0.21$ & 0.70 & $0.70 \pm 0.22$ & 0.60 & .94 \\
$\begin{array}{c}\text { (cm) } \\
\text { Inlet length } \\
\text { (cm) }\end{array}$ & $3.19 \pm 0.76$ & 3.10 & $2.97 \pm 0.63$ & 2.90 & .24 \\
$\begin{array}{c}\text { Outlet length } \\
\text { (cm) }\end{array}$ & $3.69 \pm 0.84$ & 3.55 & $3.05 \pm 0.63$ & 2.95 & .005 \\
\hline
\end{tabular}

(median 15 days); 14 of the patients had died within the first postoperative month. Additional data are shown in Table 1.

\section{Hypertrophy}

The inlet and outlet wall thicknesses were not different from those in control hearts (Table 2). The LV outlet length was significantly greater in the malformed hearts $(95 \%$ confidence interval for difference of means $0.19-1.01 ; P=.005$ by $t$ test). On microscopy, the myocyte transverse diameter did not differ from the controls in any of the sites analyzed (95\% confidence intervals for difference of means -2.1 to 1.18 for inlet, -2.29 to 0.95 for apex, and -1.37 to 1.53 for outlet, respectively; Table 3). There was no difference when comparing inlet, apex, and outlet of the $\operatorname{LV}(P=.38$ by Friedman test). In addition, the myocyte nuclear volume fraction was significantly smaller in the malformed hearts 
TABLE 3. Myocyte diameter, interstitial fibrosis and endocardial thickness in the inlet, apex, and outlet of the LV in control hearts and those with tricuspid atresia

\begin{tabular}{|c|c|c|c|c|c|c|}
\hline & \multicolumn{2}{|c|}{ Myocyte diameter $(\mu \mathrm{m})$} & \multicolumn{2}{|c|}{ Fibrosis (IFVF \%) } & \multicolumn{2}{|c|}{ Endocardial thickness $(\mu \mathrm{m})$} \\
\hline & Mean \pm SD & Median & Mean \pm SD & Median & Mean \pm SD & Median \\
\hline \multicolumn{7}{|l|}{ Inlet } \\
\hline Tricuspid atresia & $9.6 \pm 2.4$ & $9.6(25)$ & $3.8 \pm 2.8$ & $3.1(29) \dagger$ & $53.9 \pm 40.1$ & $41.8(29)^{*}$ \\
\hline Control & $10.0 \pm 2.6$ & $9.8(15)$ & $1.0 \pm 0.7$ & $1.0(23)$ & $24.0 \pm 19.7$ & $14.3(18)$ \\
\hline \multicolumn{7}{|l|}{ Apex } \\
\hline Tricuspid atresia & $9.6 \pm 2.1$ & $10.6(15)$ & $2.6 \pm 2.0$ & $2.0(25) \dagger$ & $20.8 \pm 14.7$ & $15.1(28)^{*}$ \\
\hline Control & $9.0 \pm 2.1$ & $8.7(14)$ & $0.5 \pm 0.5$ & $0.4(19)$ & $10.7 \pm 3.7$ & $9.9(19)$ \\
\hline \multicolumn{7}{|l|}{ Outlet } \\
\hline Tricuspid atresia & $8.9 \pm 1.9$ & $9.0(14)$ & $2.1 \pm 1.8$ & $1.6(22) \dagger$ & $34.1 \pm 29.0$ & $23.9(22)$ \\
\hline Control & $9.0 \pm 1.8$ & $9.4(14)$ & $0.6 \pm 0.5$ & $0.4(18)$ & $19.0 \pm 13.2$ & $14.8(16)$ \\
\hline
\end{tabular}

In parentheses are shown the numbers of cases analyzed in each comparison (tricuspid atresia versus control).

${ }^{*} P<.01$ versus control.

$\dagger P<.001$ versus control.

(median value $2.4 \%$ vs $4.4 \%, P<.001$ by Mann-Whitney test).

\section{Capillary Volume Fraction}

The myocardial capillary volume fraction was significantly smaller in hearts with tricuspid atresia than in normal control hearts (median value $6.8 \%$ vs $8.1 \%, P=.01$ by MannWhitney test).

\section{Interstitial Fibrosis}

The IFVF was significantly greater in the malformed hearts at all three sites examined (Table 3 and Figure 1, A). The LV inlet and apex were more fibrotic than the outlet in the malformed hearts but not in the control group $(P=.004$ by Friedman test). In addition, the IFVF was greater in the subendocardial halves of the ventricular wall in the inlet and apex of hearts with tricuspid atresia but not the control hearts $(P=.002$ and $P=.01$, respectively, by Wilcoxon test Figure 2).

There was a weak but significant positive correlation between IFVF and the age of the patients in the study group $\left(\mathrm{r}_{\mathrm{s}}=0.50, P=.006\right)$ but not the control group $\left(\mathrm{r}_{\mathrm{s}}=.16, P\right.$ $=.45)$.

The multivariate logistic regression model suggested that age was the only variable associated with an increased probability of occurrence of fibrosis, defined as IFVF greater than $3 \%$. The other variables included in the model (type of ventriculoarterial connection, the presence of a restrictive VSD, presence of aortic coarctation or interruption, decreased pulmonary blood flow physiology, and previous surgery) were not independent predictors of occurrence of fibrosis in the multivariate model. In fact, children older than 3 months had an 8.3 times greater probability of occurrence of fibrosis than had a younger population (odds ratio $95 \%$ confidence interval 1.47-47.22, sensitivity $71.4 \%$, specificity $76.9 \%$; Figure 3 ).

\section{Myocardial Microscars}

Myocardial microscars (Figure 1, B) were present in 7 specimens from patients aged 27 days through 50 months (median 4 months). Five were apical and 4 were restricted to the subendocardial half of the ventricular wall.

\section{Endocardial Thickness}

LV endocardial thickness was greater at the inlet and apex of the malformed hearts than in the control hearts (Table 3).

\section{Type of Ventriculoarterial Connection}

The age at death was similar between the two groups (concordant 3 days to 34 months, median 3.0 months; discordant 6 days to 50 months, median 2.3 months). There was no difference when comparisons were made between hearts with concordant and discordant ventriculoarterial connections to address the following variables: inlet wall thickness $(P=.09$ by $t$ test), inlet length $(P=.5$ by $t$ test $)$, transverse myocyte diameter $(P=.24$ by $t$ test $) ; \operatorname{IFVF}(P=$ .19 by Mann-Whitney test), and endocardial thickness $(P=$ .76 by $t$ test).

\section{Discussion}

The LV of the heart with tricuspid atresia plays a crucial role because it functions as the only pumping chamber. Few studies have addressed the myocardial structure of malformed hearts, particularly in univentricular atrioventricular connections.

\section{Hypertrophy}

In our study the LVs of the malformed hearts were dilated, without a relative increase in the wall thickness corresponding to eccentric hypertrophy, as would be expected in situations where there is predominantly volume overload. These findings are in accordance with those described by others ${ }^{6}$ in hearts with pulmonary atresia with intact ventric- 

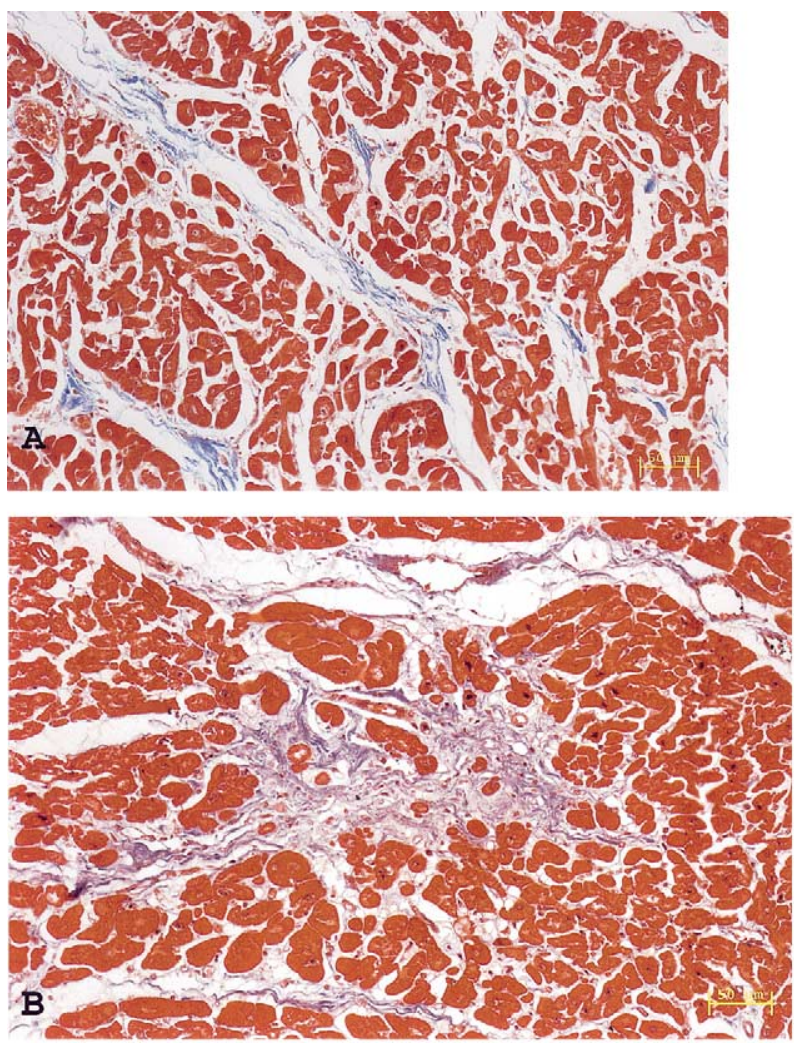

Figure 1. Photomicrographs of LV myocardium from child with tricuspid atresia (original magnification $\times 20$, Masson trichrome stain). A, Diffuse interstitial fibrosis stained in blue. B, Starshaped scar stained in blue.

ular septum, suggesting that the ratio of LV mass to enddiastolic volume may be inadequate in univentricular hearts. We did not find specimens in our material with excessive wall thickness, as demonstrated in an autopsy series of patients who died after the Fontan procedure ${ }^{9}$ and also in a clinical setting. ${ }^{10}$ This diversity in results is likely to be related to the young age of death of the patients included in our study, most younger than 1 year, and the relative paucity of cases with discordant ventriculoarterial connection in association with a restrictive VSD and subaortic stenosis, in which setting conspicuous ventricular hypertrophy is more frequent.

During the adaptive phase of elevated diastolic wall stress in volume overloads, the ratio of wall thickness to chamber radius remains constant as a result of proportional growth in the transverse and longitudinal dimensions of the myocyte. After the progression toward decompensation, there is a disproportionate lengthening of the myocytes and a dilated, thin-walled ventricular chamber. ${ }^{11}$ In this study the myocyte transverse diameter in the affected hearts did not differ from that in the control hearts. Although the longitudinal dimension could not be obtained, it is likely

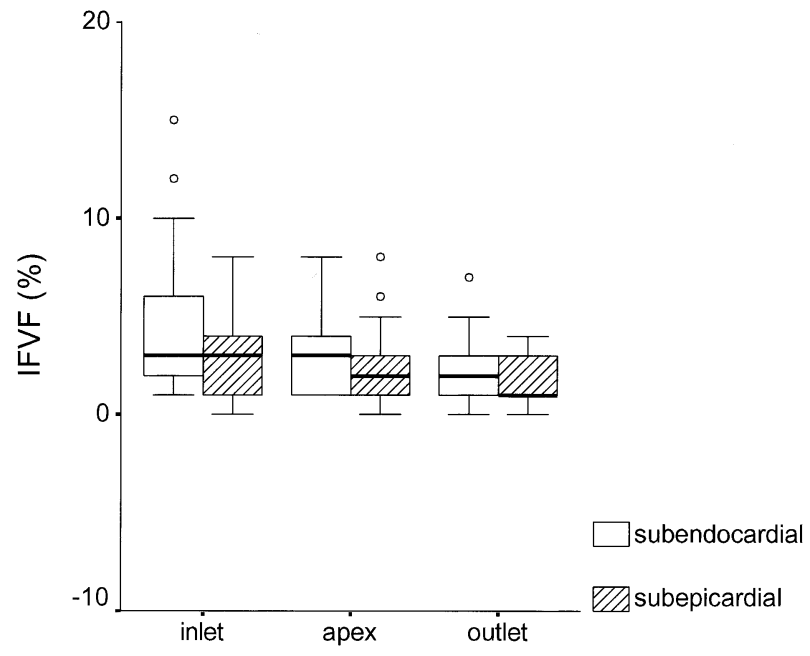

Figure 2. Box and whisker plots comparing IFVF in subendocardial and subepicardial halves of LV wall of hearts with tricuspid atresia in three sites analyzed. Boxes represent median, upper, and lower quartiles. $P$ values are $.002, .01$, and .09 , respectively, for comparisons in inlet, apex, and outlet of LV.

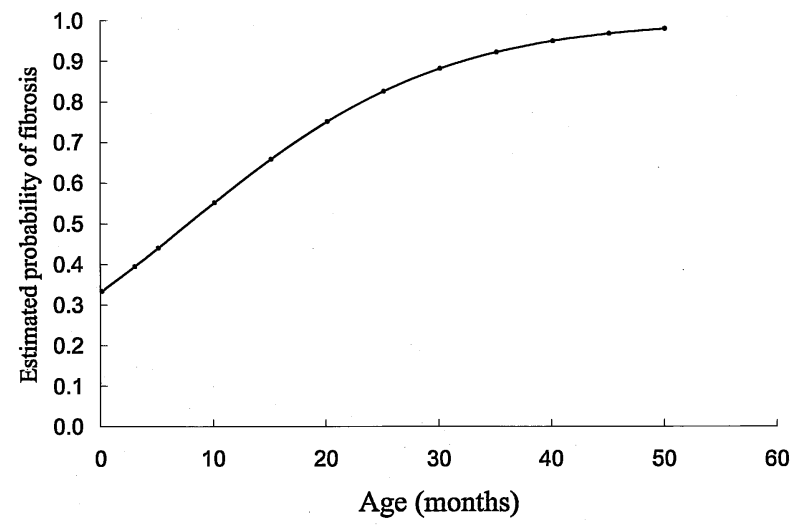

Figure 3. Estimated probability of occurrence of fibrosis in relation to age of patients.

that these patients were in the maladaptive phase of eccentric hypertrophy before death.

Experimental data have indicated that the myocyte nuclear volume increases with cellular hypertrophy. ${ }^{12}$ In addition, previous studies have demonstrated that human myocytes are prevalently mononucleated. ${ }^{13}$ The myocyte nuclear volume fraction could therefore be viewed as an index of hypertrophy and of cell number in a given area of the histologic section. The decreased nuclear volume fraction found in hearts with tricuspid atresia relative to normal control hearts is in agreement with a predominantly longitudinal growth of the myocytes (eccentric hypertrophy). However, we could not establish a parallel to the hypertro- 
phic process, either because one does not exist or because of the relatively small number of cases analyzed.

\section{Coronary Capillaries}

The myocardial capillary network expands proportionally during physiologic growth ${ }^{14}$ but may be inadequate under conditions of hemodynamic overload, increasing the potential for ischemic damage in cardiac hypertrophy. Evidence from animal and human studies indicates that capillary growth in pressure overload hypertrophy does not fully compensate for the increase in ventricular mass, ${ }^{15}$ although limited capillary growth may occur in young patients ${ }^{16}$ and during adaptation to a prolonged pressure overload. ${ }^{17}$ In contrast, several experimental studies in volume overload models suggest that the adaptation of the myocardial capillary network is proportional to the degree of hypertrophy. ${ }^{18}$ Experimental data on the myocardial capillary supply adaptation in response to hypoxia are controversial. ${ }^{15}$ Yet hypoxia is considered to be an effective stimulus for growth of endothelial cells. ${ }^{15}$ The finding of a decreased capillary volume fraction in our cases might indicate a greater vulnerability of these hearts to ischemia.

\section{Extracellular Matrix}

Hearts with tricuspid atresia showed a higher proportion of fibrous tissue than normal since the first weeks of life, showing a positive correlation with increasing age. The multivariate logistic regression model indicated that infants older than 3 months had an 8.3 times greater probability of occurrence of fibrosis than had younger infants, supporting the view that the myocardium may be inherently abnormal. ${ }^{4,5}$

It has been well documented that pressure overload cardiac hypertrophy is accompanied by an increased accumulation of collagen in the myocardium. ${ }^{19,20}$ Fibrosis of the heart accompanies chronic elevations in circulating effector hormones of the renin-angiotensin-aldosterone system, when heart failure or unilateral renal artery stenosis impairs renal perfusion. ${ }^{20}$ In contrast, most of the published experimental data have indicated that interstitial collagen accumulation does not change in response to induced volume overload. $^{21,22}$

Much attention is currently focused on the role of apoptosis in the pathogenesis of cardiac disease. Apoptosis has been implicated in a wide range of models, such as ischemia and myocardial infarction, pressure overload hypertrophy, cardiac rejection, and dilated cardiomyopathy. ${ }^{23}$ It may be a contributing phenomenon in the pathologic remodeling in hearts with tricuspid atresia.

The morphometric method permitted the discrimination of the fibrous deposition across the ventricular wall. Hoyt and colleagues ${ }^{7}$ compared the quantification of collagen in histologic sections stained with Masson trichrome, per- formed with a computer-assisted method similar to ours, with those obtained from a hydroxyproline assay. The percentage of collagen from analysis of histologic sections strongly correlated with the hydroxyproline level, with excellent intraobserver and interobserver concordance. Results were unaffected by variations in histochemical staining.

Our study showed a greater deposition of fibrous tissue in the subendocardial half of the LV wall, following the pattern found in concentric hypertrophy, ${ }^{24}$ dilated cardiomyopathy, ${ }^{25}$ and ventricular remodeling after myocardial infarction. ${ }^{26}$ This finding is not in agreement with observations by $\mathrm{Ho}$ and colleagues ${ }^{4}$ in hearts with tricuspid atresia. Experimental studies of physiologic growth have demonstrated a relatively lower capillary luminal volume in the subendocardial layer, indicating a greater vulnerability of this region to ischemic injury. ${ }^{27}$ In addition, the myocardial tissue pressure and oxygen demands are greater in the subendocardial portion of the wall. Thus the disturbance of oxygen supply and demand may result in chronic tissue injury and subendocardial fibrosis.

This analysis demonstrated regional variations in the fibrous deposition, with the LV inlet and apex being more fibrotic than the outlet in malformed hearts. This finding could explain, at least in part, regional ventricular wall motion abnormalities found in patients with univentricular hearts before and after stages of Fontan reconstruction. ${ }^{28,29}$

The comparison according to the type of ventriculoarterial connections did not show any significant difference in the content of fibrous tissue. This finding is in conformity with data published by Ho and colleagues, ${ }^{4}$ possibly reflecting the dominant role of the LV.

The functional consequences of collagen remodeling have been extensively demonstrated. ${ }^{30}$ It seems clear that both systolic and diastolic properties may be affected. Clinical trials with pharmacologic interventions directed toward collagen remodeling in such malformed hearts should be addressed in future studies.

\section{Conclusion}

On the whole, our findings in hearts with tricuspid atresia are more likely to represent a chronically induced volume overload in the presence of ischemia than to represent a typical model of volume overload. Moreover, the pathologic insult occurs in parallel with the expansion of cardiac mass in a growing child. What remains to be clarified is why the capillary network seems not to develop properly in the presence of a strong potential stimulus for angiogenesis (hypoxia).

We thank Ms Creusa Maria Roveri dal Bó for assistance with statistical analysis and Professor Antonio Augusto Barbosa Lopes for his valuable suggestions. 


\section{References}

1. Fontan F, Kirklin JW, Fernandez G, Costa F, Naftel DC, Tritto F, et al. Outcome after a "perfect" Fontan operation. Circulation. 1990;81: 1520-36.

2. Gentles TL, Mayer JE Jr, Gauvreau K, Newburger JW, Lock JE, Kupferschmid JP, et al. Fontan operation in five hundred consecutive patients: factors influencing early and late outcome. J Thorac Cardiovasc Surg. 1997;114:376-91.

3. Cohen AJ, Cleveland DC, Dyck J, Poppe D, Smallhorn J, Freedom RM, et al. Results of the Fontan procedure for patients with univentricular heart. Ann Thorac Surg. 1991;52:1266-70.

4. Ho SY, Jackson M, Kilpatrick L, Smith A, Gerlis LM. Fibrous matrix of ventricular myocardium in tricuspid atresia compared with normal heart: a quantitative analysis. Circulation. 1996;94:1642-6.

5. Sanchez-Quintana D, Climent V, Ho SY, Anderson RH. Myoarchitecture and connective tissue in hearts with tricuspid atresia. Heart. 1999;81:182-91.

6. Akiba T, Becker AE. Disease of the left ventricle in pulmonary atresia with intact ventricular septum: the limiting factor for long-lasting successful surgical intervention? J Thorac Cardiovasc Surg. 1994;108: $1-8$.

7. Hoyt RH, Ericksen E, Collins SM, Skorton DJ. Computer-assisted quantitation of myocardial fibrosis in histologic sections. Arch Pathol Lab Med. 1984;108:280-3.

8. Weibel ER, Gomez DM. A principle for counting tissue structures on random sections. J Appl Physiol. 1962;17:343-8.

9. Caspi J, Coles JG, Rabinovich M, Cohen D, Trusler GA, Williams WG, et al. Morphological findings contributing to a failed Fontan procedure: twelve-year experience. Circulation. 1990;82(5 Suppl): IV177-82.

10. Malcic I, Sauer U, Stern H, Kellerer M, Kuhlein B, Locher D, et al. The influence of pulmonary artery banding on outcome after the Fontan operation. J Thorac Cardiovasc Surg. 1992;104:743-7.

11. Gerdes AM, Clark LC, Capasso JM. Regression of cardiac hypertrophy after closing an aortocaval fistula in rats. Am J Physiol. 1995; 268(6 Pt 2):H2345-51.

12. Gerdes AM, Liu Z, Zimmer HG. Changes in nuclear size of cardiac myocytes during the development and progression of hypertrophy in rats. Cardioscience. 1994;5:203-8.

13. Olivetti G, Cigola E, Maestri R, Corradi D, Lagrasta C, Gambert SR, et al. Aging, cardiac hypertrophy and ischemic cardiomyopathy do not affect the proportion of mononucleated and multinucleated myocytes in the human heart. J Mol Cell Cardiol. 1996;28:1463-77.

14. Anversa P, Ricci R, Olivetti G. Coronary capillaries during normal and pathological growth. Can J Cardiol. 1986;2:104-13.

15. Hudlicka O, Brown MD. Postnatal growth of the heart and its blood vessels. J Vasc Res. 1996;33:266-87.

16. Rakusan K, Flanagan MF, Geva T, Southern J, Van Praagh R. Morphometry of human coronary capillaries during normal growth and the effect of age in left ventricular pressure-overload hypertrophy. Circulation. 1992;86:38-46.

17. Tomanek RJ, Wessel TJ, Harrison DG. Capillary growth and geometry during long-term hypertension and myocardial hypertrophy in dogs. Am J Physiol. 1991;261(4 Pt 2):H1011-8.

18. Legault F, Rouleau JL, Juneau C, Rose C, Rakusan K. Functional and morphological characteristics of compensated and decompensated cardiac hypertrophy in dogs with chronic infrarenal aorto-caval fistulas. Circ Res. 1990;66:846-59.

19. Schwartzkopff B, Frenzel H, Dieckerhoff J, Betz P, Flasshove M, Schulte HD, et al. Morphometric investigation of human myocardium in arterial hypertension and valvular aortic stenosis. Eur Heart J. 1992;13(Suppl D):17-23.

20. Weber KT, Sun Y, Guarda E, Katwa LC, Ratajska A, Cleutjens JP, et al. Myocardial fibrosis in hypertensive heart disease: an overview of potential regulatory mechanisms. Eur Heart J. 1995;16(Suppl C):24-8.

21. Weber KT, Pick R, Silver MA, Moe GW, Janicki JS, Zucker IH, et al. Fibrillar collagen and remodeling of dilated canine left ventricle. Circulation. 1990;82:1387-401.

22. Namba T, Tsutsui H, Tagawa H, Takahashi M, Saito K, Kozai T, et al. Regulation of fibrillar collagen gene expression and protein accumulation in volume-overloaded cardiac hypertrophy. Circulation. 1997; 95:2448-54.

23. Davies MJ. Apoptosis in cardiovascular disease. Heart. 1997;77:498501.

24. Lund O, Kristensen LH, Baandrup U, Hansen OK, Nielsen TT, Emmertsen $\mathrm{K}$, et al. Myocardial structure as a determinant of pre- and postoperative ventricular function and long-term prognosis after valve replacement for aortic stenosis. Eur Heart J. 1998;19:1099-108.

25. Unverferth DV, Baker PB, Swift SE, Chaffee R, Fetters JK, Uretsky $\mathrm{BF}$, et al. Extent of myocardial fibrosis and cellular hypertrophy in dilated cardiomyopathy. Am J Cardiol. 1986;57:816-20.

26. Michel JB, Nicoletti A, Arnal JF. Left ventricular remodelling following experimental myocardial infarction. Eur Heart J. 1995;16(Suppl I):49-57.

27. Anversa P, Capasso JM, Ricci R, Sonnenblick EH, Olivetti G. Morphometric analysis of coronary capillaries during physiologic myocardial growth and induced cardiac hypertrophy: a review. Int J Microcirc Clin Exp. 1989;8:353-63.

28. Akagi T, Benson LN, Williams WG, Freedom RM. Regional ventricular wall motion abnormalities in tricuspid atresia after the Fontan procedure. J Am Coll Cardiol. 1993;22:1182-8.

29. Fogel MA, Gupta KB, Weinberg PM, Hoffman EA. Regional wall motion and strain analysis across stages of Fontan reconstruction by magnetic resonance tagging. Am J Physiol. 1995;269(3 Pt 2):H113252.

30. Conrad CH, Brooks WW, Hayes JA, Sen S, Robinson KG, Bing OH. Myocardial fibrosis and stiffness with hypertrophy and heart failure in the spontaneously hypertensive rat. Circulation. 1995;91:161-70. 